\title{
Performance of a Checkerboard Liner with Uncertain Impedances
}

\author{
J. H. Robinson, and W. R. Watson ${ }^{\dagger}$ \\ NASA Langley Research Center, Hampton, Virginia 23681-2199, USA
}

\begin{abstract}
The current fleet of large commercial aircraft has successfully achieved FAA noise certifications because of, in part, the successful application of uniform passive duct liner treatments to control engine system noise. One goal of NASA's engine system noise reduction program is to develop technologies to improve the sound absorbing properties of duct liner treatments so that they remain effective in modern turbo fan engines. One such technology being studied is checkerboard or periodic axially and circumferentially segmented liners. A preliminary assessment of the potential of this technology was conducted by applying uncertainties associated with manufacturing, installation, source structure, and tonal frequency to a liner developed using deterministic design methods and generating a measure of improvement with respect to a uniform liner subjected to the same uncertainties. Deterministic design and analysis of the candidate checkerboard liner showed that it obtains a $1.5 \mathrm{~dB}$ per duct aspect ratio improvement in liner attenuation over a similarly designed uniform liner. When uncertainties in liner impedances, source structure, and frequency are considered, the performance of the checkerboard liner drops off dramatically. The final results of this paper show that the candidate checkerboard liner has a less than 25 percent chance of outperforming the uniform liner when moderate levels of uncertainty are considered. It is important to note that this study did not include the effects of mean flow on liner performance and, more important to note, that as a gradient based optimization process was used to design the checkerboard liner, it is almost certain that a global optimal design was not found for the candidate checkerboard liner. Had it been possible to find a better deterministically performing checkerboard liner, the probability that this candidate liner would outperform the uniform liner would certainly have been higher.
\end{abstract}

\section{Nomenclature}

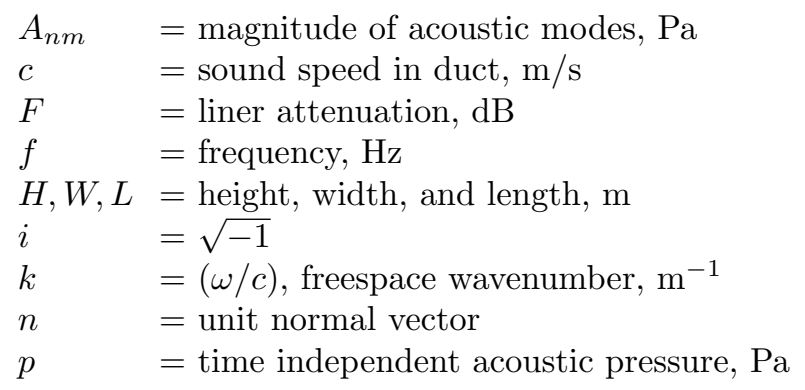

*Aerospace Engineer, Aeronautics Systems Analysis Branch, Systems Analysis and Concepts Directorate, Mail Stop 455. Member of AIAA.

${ }^{\dagger}$ Senior Research Scientist, Computational Aerosciences Branch, Research \& Technology Directorate, Mail Stop 128. Senior member of AIAA. 


$\begin{array}{ll}P & =\text { probability } \\ R, \chi & =\text { normalized acoustic resistance and reactance } \\ t & =\text { dimensional time, } \mathrm{s} \\ x, y, z & =\text { transverse, spanwise, and axial coordinates } \\ \zeta & =R+i \chi, \text { normalized acoustic impedance } \\ \nabla^{2} & =\text { three-dimensional Laplace operator, } \mathrm{m}^{-2} \\ \Delta & =\text { increment of attenuation, } \mathrm{dB} \\ \theta_{n m} & =\text { phase of acoustic modes, radians } \\ \rho & =\text { ambient density, } \mathrm{kg} / \mathrm{m}^{3} \\ \rho c & =\text { characteristic impedance of air, } \mathrm{kg} \mathrm{s}^{-1} \mathrm{~m}^{-2} \\ \omega & =2 \pi f, \text { angular frequency, } \mathrm{s}^{-1} \\ \text { Subscripts: } & \\ e x i t & =\text { exit plane } \\ u, c & =\text { uniform and checkerboard liner quantity } \\ I & =\text { the } I \text { th impedance segment } \\ n, m & =\text { mode order in cross duct directions } \\ s & =\text { source plane }\end{array}$

\section{Introduction}

The concept of the checkerboard liner is generally attributed to Mani ${ }^{1}$ who having shown experimentally that a circumferentially segmented liner could provide better attenuation than a uniform liner, proposed that a liner that is segmented both axially and circumferentially (i.e., the checkerboard liner) may have potential for additional improvements. The paucity of research on the sound attenuation produced by checkerboard liners is due to a lack of fully 3-D analysis codes for modeling the liner attenuation within the duct. To date, no experimental and only four analytical studies ${ }^{2-5}$ of sound attenuation produced by checkerboard liners have been reported. Howe ${ }^{2}$ compared sound attenuation produced by uniform, axially segmented, circumferentially segmented, and checkerboard liners. The primary conclusion of that study was that the checkerboard liner offers the best means of increasing the attenuation over and above that of a uniformly lined duct. The fact that Howe ${ }^{2}$ used a very restricted theoretical analysis (the kinetic theory of gases) to model liner attenuation, however, clouded the results of this study, and, Howe did not have liner optimization studies to support the conclusions of his paper. Watson et al. ${ }^{3}$ conducted a study of three optimization techniques (iterative contour deformation, Davidon-Fletcher-Powell, genetic algorithm) for optimizing the attenuation produced by checkerboard liners. It was concluded that the genetic algorithm was the most reliable of the optimization techniques due to its ability to handle multiple local optima generated by the checkerboard liner. In a subsequent study, Watson et al. ${ }^{4}$ combined the genetic algorithm with a numerical solution to the Helmholtz equation to demonstrate that an optimized 8-segment checkerboard liner gives considerably more attenuation than an optimum uniform or 4-segmented checkerboard liner. However, central processor unit (CPU) time and memory constraints and the occurrence of multiple local optima limited these results to a single frequency. Watson et al. ${ }^{5}$ extended this analysis to multiple frequencies for the same configuration. The primary conclusion of the study is that an optimized checkerboard liner attenuates substantially more sound than an optimized uniform liner, and it has a broader off-design performance. Relative to the uniform liner, the checkerboard liner tends to be more effective at the higher frequencies.

During the design optimization and assessment studies of Watson et al., ${ }^{3-5}$ they observed that the checkerboard liner appeared significantly more sensitive to variations in the impedance values than did the uniform. This trend was also observed in the initial studies involving axially segmented, circumferentially segmented, and checkerboard liners. This is significant in that the values reported in many of the studies for liner impedances had significant digits greater than the manufactured tolerances of this type of liner. It 
was also apparent that the structure of the source model strongly influenced the resulting distribution of impedances. This was most evident in the 2- and 4-segmented liner studies in which it was anticipated that some mirror image liner designs would appear. An investigation into their absence concluded that the source mode structure skewed the anticipated results. A further review of the literature found this observation had been previously reported.

In the $70 \mathrm{~s}$, the axially segmented liner was studied extensively. Motsinger ${ }^{6} \mathrm{Kraft}^{7}{ }^{7}$ and Lester ${ }^{8}$ performed some studies of particular interest to this investigation. However, the axially segmented lining configuration was not implemented in the current generation of aircraft engines because their optimum design requires an accurate description of the modal distribution of acoustic energy in the sound source. The problem of determining the modal amplitude and relative phasing of source modes within wide chord fan engine ducts is extremely difficult.

Baumeister, ${ }^{9}$ who studied the performance of multielement liners, provided a further indication of the dependence of optimum liner impedances on source structure. His observations were similar to those of Watson, concluding that multielement liners are most advantageous at high frequencies and did, theoretically, outperform uniform liners. They also observed that multielement liners suffered from large degradation in performance in response to changes in the assumed input source structure.

The work presented in this paper was motivated by the need to provide an assessment of what performance gains may be attainable in an actual experiment or in service conditions by using checkerboard liners. The primary uncertainty that is the focus of this paper is the uncertainty in liner impedance associated with the manufacturing and installation of these liners in scale model engines and test facilities. Previous studies of the performance of checkerboard liners did not take into account these uncertainties. The level of variability in liner impedances is taken to be plus or minus one-tenth of a $\rho c$, about the nominal liner impedance value for this investigation. This value is more of a goal of NASA's engine noise reduction systems program than a true reflection of the current state of the art. Previous publications ${ }^{9}$ have suggested a variance in impedance of approximately twice this value. Other sources of uncertainty associated with operational conditions are assumed to be independent of liner uncertainties and to be taken into account through applying uncertainties to the source structure and frequency. The measure of the influence of these uncertainties on the performance of checkerboard liners is presented with respect to the performance of a uniform liner subjected to the same level of uncertainties in manufacturing and installation, operating conditions, and source structure. The remainder of this paper is broken into sections covering various aspects of this assessment. The next section of this paper discusses the problem formulation and solution methodology. The third section will discuss the uncertain variables, assessment metric, and procedure. The final section will present results with respect to specific variables. A brief discussion of the results and references will follow. Thus, this study will assess the advantages and disadvantages of a periodic checkerboard liner configuration over the currently installed uniform liners with uncertainties.

\section{Problem Formulation}

Both the uniform and checkerboard liners are evaluated using a geometry corresponding to that of the grazing incidence tube in the NASA Langley Research Center Flow Impedance Test Facility. This geometry and the selected frequency range were chosen so that experimental tests could be conducted at a later date to corroborate the computed results. Figure 1 gives a schematic of the 3-D, zero flow duct, and right-handed coordinate system used to predict the liner attenuation properties. The checkerboard liner is axially centered between the source and exit plane. The upper and two side walls of the duct are rigid. Acoustic waves are propagated from left to right across the surface of the liner and into a termination section of duct that is designed to be nonreflecting to plane acoustic waves. The test section is $0.812 \mathrm{~m}$ long and has a cross section of $0.051 \mathrm{~m} \mathrm{x} 0.051 \mathrm{~m}$. The source and exit planes are located in hard wall sections of the duct, $0.203 \mathrm{~m}$ from the leading and trailing edges of the liner, respectively. 


\section{A. Governing Equations, Solution, and Objective}

The mathematical problem is to find the solution to the Helmholtz equation subject to the liner impedance boundary condition along the periphery of the duct, the exit impedance boundary condition at the duct exit, and a known sound source condition at the source plane $(z=$ $0)$. The Helmholtz equation is

$$
\nabla^{2} p+k^{2} p=0
$$

where a time convention of the form $e^{i \omega t}$ is assumed. The boundary condition along the rigid-wall portions of the duct is equivalent to the requirement that the gradient of acoustic pressure normal to the wall vanishes, that is

$$
\frac{\partial p}{\partial n}=0
$$

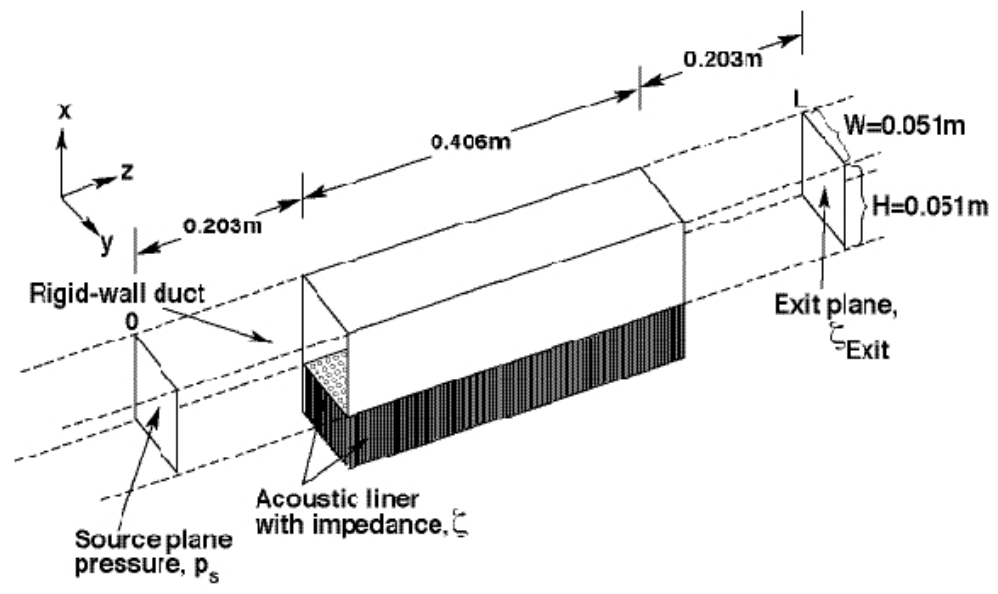

Figure 1. Schematic of grazing incidences impedance tube and liner configuration.

where $n$ is the unit normal vector to the duct wall and is taken as positive when pointing into the fluid. At the duct exit $(z=L)$, the ratio of acoustic pressure to the axial component of acoustic particle velocity must equal the exit acoustic impedance. When expressed in terms of the acoustic pressure, this boundary condition is

$$
\frac{\partial p}{\partial z}=\frac{-i k p}{\zeta_{\text {exit }}}
$$

A dimensional termination impedance of $\rho c\left(\zeta_{\mathrm{e}}=1\right)$ is used as a reasonable proxy impedance for all modes. It is not possible to know a priori the resultant impedance of some arbitrary superposition of modes incident upon the computational boundary plane. This exit impedance will not lead to an exact simulation of the physical termination of the grazing incidence tube because it may not allow higher order modes to transmit through the computational boundary properly. However, it is a more accurate representation of the grazing incidence tube termination than a nonreflecting boundary condition.

The wall lining is assumed to be locally reacting, so that the liner boundary condition is

$$
\frac{\partial p}{\partial n}=\frac{i k p}{\zeta}
$$

At the source plane $(z=0)$ of the duct, the sound source pressure, $p_{s}$, is known. Therefore, the sound source boundary condition is

$$
p=p_{s}
$$

Eqs. (1-5) form a well posed boundary value problem that one can solve to determine uniquely the acoustic pressure field in the duct. An exact analytical solution for this field is not available for a general input data set; therefore, a numerical method is used to obtain the solution for this field.

In previous publications, this system of equations was solved using a research grade finite element program. This program utilized the scientific library on Silicon Graphics, Inc. computers. In this investigation the solution methodology was transferred to the commercial code Actran. ${ }^{10}$ This code directly calculated the radiated power out the end of the finite length duct. A duct with rigid walls was used as a reference point from which to calculate the objective function. 
Watson et al. ${ }^{4}$ used the reduction in sound power from the source to the exit of the duct as their objective function. This function was maximized to determine the optimal attenuation of the liner. This investigation also uses this measure of the reduction in sound power as the primary measure of performance for individual liners. This function is normalized as before with respect to the duct and liner dimensions and has units of decibels per $\left(L_{2}-L_{1}\right) / H$. The radiated sound power is calculated directly by the analysis program Actran ${ }^{10}$ and no further post processing is necessary other than normalizing the result.

\section{Uncertainty assessment}

The desired metric for the assessment of the liner performance under uncertainty can be stated as the probability that the attenuation of the checkerboard liner is greater than the attenuation of the uniform liner by an arbitrary increment $\Delta$. This increment, along with the probability of attaining it, can be used to assess the merits of further exploring this concept subjectively. Mathematically this probability is stated as

$$
P\left(F_{c}\left(\zeta_{I}, \theta_{n m}, \omega\right)>F_{u}\left(\zeta_{u}, \theta_{n m}, \omega\right)+\Delta\right)
$$

where $\zeta_{I}, \zeta_{u}, \theta_{n m}$, and $\omega$ are independent random variables quantifying uncertainties in the impedance of the $I^{\text {th }}$ liner segment, impedance of the uniform liner, modal source phase, and source frequency, respectively. This paper discusses each of these separately in successive sections. The probability stated in Eq. (6) is calculated from the statistics of the uniform and checkerboard liner attenuations as

$$
P\left(F_{c}>F_{u}+\Delta\right)=\int_{-\infty}^{+\infty} \int_{F_{u}+\Delta}^{+\infty} p d f\left(F_{c}, F_{u}\right) d F_{c} d F_{u}
$$

where $p d f\left(F_{c}, F_{u}\right)$ is the joint probability density of the liner attenuations. Since $F_{c}$ and $F_{u}$ are independent, Eq. (7) can be written as

$$
P\left(F_{c}>F_{u}+\Delta\right)=\int_{-\infty}^{+\infty}\left[1-c d f\left(F_{u}+\Delta\right)\right] p d f\left(F_{u}\right) d F_{u}
$$

where $c d f\left(F_{u}+\Delta\right)$ is the cumulative distribution function for the checkerboard liner evaluated at $F_{c}=$ $F_{u}+\Delta$. The probability and cumulative probability distribution functions are obtained by direct Monte Carlo simulation, and the integration in Eq. (8) is carried out numerically.

\section{A. Modal Source Model with Uncertainties}

A modal pressure distribution appropriate for a multimodal noise source located in a rigid-wall section of duct,

$$
p(x, y, 0)=\sum_{n=0}^{N} \sum_{m=0}^{M} A_{n m} e^{i \theta_{n m}} \cos (n \pi y / W) \cos (m \pi x / H)
$$

is used to model the sound source. The upper limits of summation are only taken over the cut-on modes, which were assumed to have unit amplitude and zero phase (i.e., $A_{n m}=1$ and $\theta_{n m}=0$ ) in previous investigations. ${ }^{5}$ In this investigation, the amplitude, $A_{n m}$, is again assumed to have unit amplitude for all modes; however, more of the phase of each mode, $\theta_{n m}$, is considered uncertain. The phase will be allowed to vary from $-\pi$ to $\pi$ with all angles being equallikely. Note the use of Eq. (9) in this and the previous investigations contains an assumption that the rigid-wall duct source does not vary appreciably with the introduction of the liner into the duct. 


\section{B. Liner Impedances with Uncertainties}

The uncertainty in liner impedance arises primarily from manufacturing and installation considerations. Previous investigations that have considered uncertainty have used values of $0.2 \rho c$. This investigation uses the value of $0.1 \rho c$. This value is not representative of the current state of manufacturability but is a reflection of the goal of NASA's engine noise reduction systems program. As in previous publications, the resistance and reactance of the liner segments will be assumed to be independent random variables. The mean value for these variables will be the values that were obtained from the deterministic optimization study performed at $7 \mathrm{kHz}$. These results will be revisited and presented in the first section of the result section of this paper.

\section{Frequency Dependence and Uncertainties}

The variation in frequency arises quite naturally in experimental and real world situations. The magnitude of variation of frequency is assumed only to be a few percent. In this investigation, the mean frequency is that for which the liner impedances were optimized. Thus, the the frequency is assumed to be Gaussian distributed with the mean value of $7 \mathrm{kHz}$ and a standard deviation of $180 \mathrm{~Hz}$. This frequency range was also chosen such that the structure of the source would not change over this range. That is, no modes would be cut-on or cut off in this frequency range. The frequency dependence of the liner impedances must also be modeled. Watson et al. ${ }^{5}$ used a suitable model for this facility in their previous investigation. This model takes the form

$$
\zeta_{I}=R_{I}+i \chi_{I}=R_{I}+i \cot \left(k d_{I}\right)
$$

where $R_{I}$ and $\chi_{I}$ are the resistance and the reactance, respectively and are taken to be specified at the design frequency. The resistance is assumed independent of frequency, and the reactance is assumed to vary as the cotangent of frequency. The value $d_{I}$ in Eq. (10) is the depth of the liner. This model is consistent with the experimental findings of Gallman and Kunze ${ }^{11}$ for a similar facility.

\section{Monte Carlo Technique}

A Monte Carlo technique was used to estimate the probability and cumulative probability density functions of the liner attenuation. The commercial software package iSIGHT ${ }^{12}$ was used for this process. With variations in impedances, frequency, and modal phase, the distribution of the uniform liner attenuation was estimated using approximately 400 samples. The estimate for the segmented liner with four independent impedances, as well as the uncertainties in frequency and phase, used 1000 samples. The criteria for the number of samples was subjective in that only the minimum number of samples to accurately capture the distribution functions for each of the individual variables were generated. The convergence of the mean and variance of the attenuations were, however, verified. This investigation did not apply formal methods to the sampling. The distribution functions were estimated from the Monte Carlo results using standard histogram methods with 50 equally spaced bins. These results will also be presented in the second section of the results section of this paper.

\section{Results}

The result section of this paper is broken into three sections. The first section will discuss the deterministic optimization results and examine briefly the characteristics of the acoustic field inside the lined ducts with respect to the unlined duct. The second section will discuss the results from the Monte Carlo simulation using the liner impedances, source phase, and frequency as sources of uncertainty. The final section will present

the results from the assessment and present the trade-off between liner performance and the likelihood of obtaining the performance. For clarity the geometry of the duct and liner arrangement is repeated here. Figure 1 gives a schematic of the 3-D, zero flow duct, and right-handed coordinate system. The checkerboard 
liner is axially centered between the source and exit plane. The acoustic waves are propagated from left to right across the surface of the liner. The test section is $0.812 \mathrm{~m}$ long and has a cross section of $0.051 \mathrm{~m} \mathrm{x}$ $0.051 \mathrm{~m}$. The source and exit planes are located in hard wall sections of duct, $0.203 \mathrm{~m}$ from the leading and trailing edges of the liner, respectively. The radiated sound power is calculated at the exit plane. The cut-on frequencies for the rigid-wall duct modes of interest and the next higher modes are presented in Table 1

Table 1. Mode Cut-On Frequencies

\begin{tabular}{lc} 
Mode Number $(\mathrm{n}, \mathrm{m})$ & Frequency (hertz) \\
\hline$(0,0)$ & $\mathrm{N} / \mathrm{A}$ \\
$(1,0)$ & 3346.457 \\
$(0,1)$ & 3346.457 \\
$(1,1)$ & 4732.602 \\
$(0,2)$ & 6692.914 \\
$(2,0)$ & 6692.914 \\
$(1,2)$ & 7482.905 \\
$(2,1)$ & 7482.905 \\
\hline \hline
\end{tabular}

The orientation of the impedances in the checkerboard liner are shown in Fig. 2. Impedances $\zeta_{1}$ and $\zeta_{2}$ are closest to the source plane. The numerical model of the duct is comprised of 90,457 nodes using 79,872 linear hexahedral finite elements. There are 17 finite element nodes in the cross duct directions and 313 nodes along the length of the duct. This is the identical numerical model used in previous investigations.

\section{A. Optimization Results}

This paper presents limited optimization results with respect to those presented in previous publications. During the preparation of this paper and conduction of the studies outlined, an error in the source structure of the previous publications was detected. There were insufficient time and resources available to rerun all of the deterministic optimization results using a genetic algorithm. The deterministic optimization procedure used in this investigation was gradient based and, subsequently, could not be guaranteed to find global optima particularly in the case of the checkerboard liner. The impedances tabulated in the previous studies were, however, used as starting points for the optimization in this study. Table 2 presents the impedance values obtained in this investigation
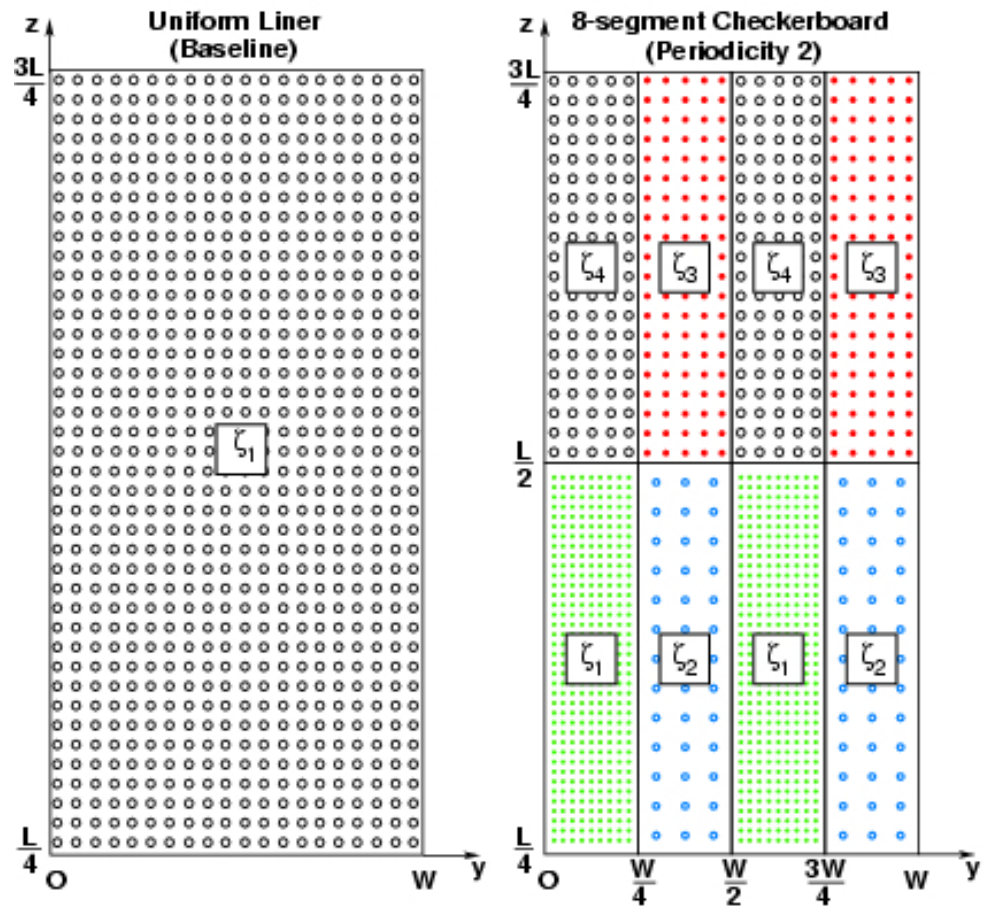

Figure 2. Schematic of uniform and periodic checkerboard liners. 
at design frequency of $7 \mathrm{kHz}$ with all modes of equal amplitude and zero relative phase. In this table the uniform liner impedance is $\zeta_{u}$ and $\zeta_{1}, \zeta_{2}, \zeta_{3}$ and $\zeta_{4}$ are the impedances of the checkerboard liner.

Table 2. Impedance Values From Optimization

\begin{tabular}{lcc} 
Segment & impedance $\rho c$ & linerdepth $(\mathrm{mm})$ \\
\hline$\zeta_{u}$ & $2.37-2.35 i$ & 3.09 \\
$\zeta_{1}$ & $3.46-3.70 i$ & 2.04 \\
$\zeta_{2}$ & $0.62-3.25 i$ & 2.31 \\
$\zeta_{3}$ & $2.25-0.42 i$ & 9.04 \\
$\zeta_{4}$ & $1.48-0.81 i$ & 6.88 \\
\hline \hline
\end{tabular}

The performances obtained by this process were $2.28 \mathrm{~dB}$ per duct aspect ratio for the uniform liner and $3.74 \mathrm{~dB}$ per duct aspect ratio for the checkerboard liner. Table 2 also presents values for the liner depth obtained from Eq. (10). No attempt was made to constrain these values to physically realizable quantities. It is clear from this table that most of these liners are nonphysical, having depths less than the thickness of typical liner face sheets. This practice, however, is consistent with previous related publications. The process of applying such a constraint would not have significantly complicated the design and assessment process. The different acoustic fields inside the uniformly, checkerboard, and unlined ducts are shown in Fig. 3, respectively. The unlined duct is presented for completeness and does show that the acoustic field is correctly resolved. It is also important to note the strength of reflections off the termination.

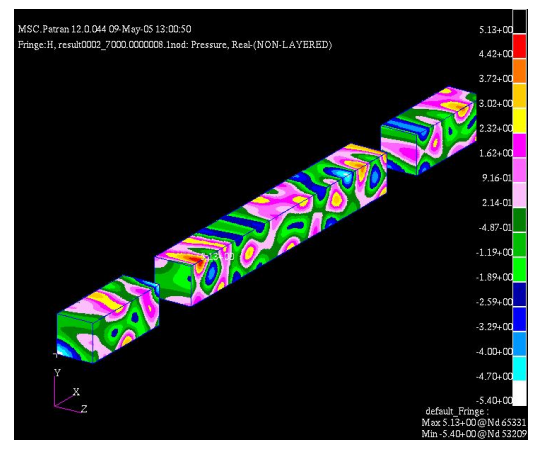

(a) No liner.

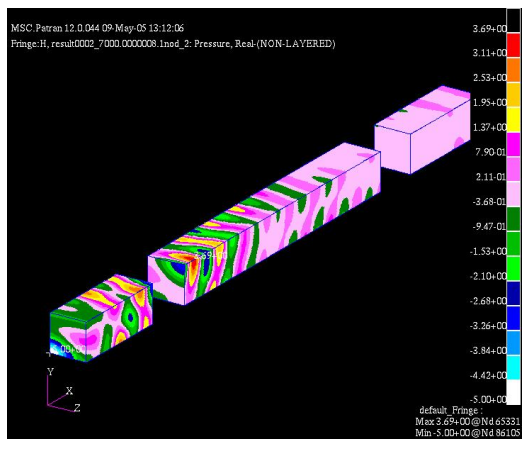

(b) Uniform liner.

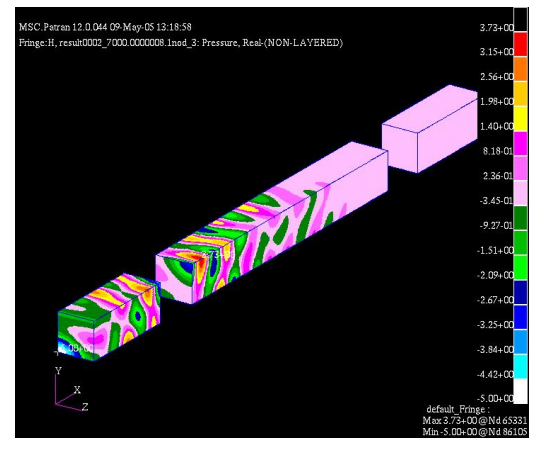

(c) Checkerboard liner.

Figure 3. Comparison of real part of pressure in duct with no liner, uniform liner, and checkerboard liner at $7 \mathrm{kHz}$.

\section{B. Monte Carlo Results}

Monte Carlo simulations were conducted on the uniformly and checkerboard lined ducts about the deterministic optimum solution. The uncertainties used were those previously stated for liner impedance, source phase, and frequency. For the uniform duct, this resulted in eight uncertain variables; and for the checkerboard lined duct, 14 uncertain variables. This investigation used 1000 random samples for the checkerboard liner and 423 random samples for the uniformly lined duct. The odd number of samples for the uniformly lined duct is a product of a premature termination to the run. The simulations did appear to converge; however, no formal methods were applied to the sampling process. For completeness the distributions of the 
uncertain variables are shown in Fig. 4 for the uniformly lined duct and in Fig. 5 for select variables of the checkerboard lined duct. It is important to note that the frequency, being Gaussian distributed, does span cutoff frequencies of several modes both above and below the design frequency.

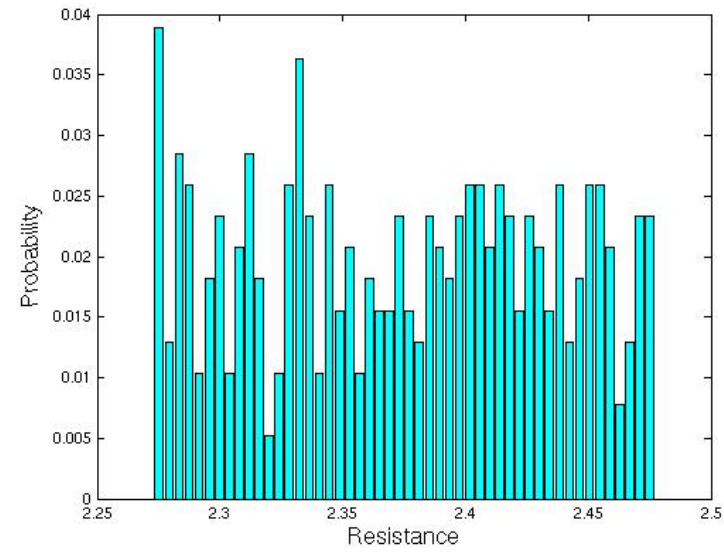

(a) Resistance Distribution.

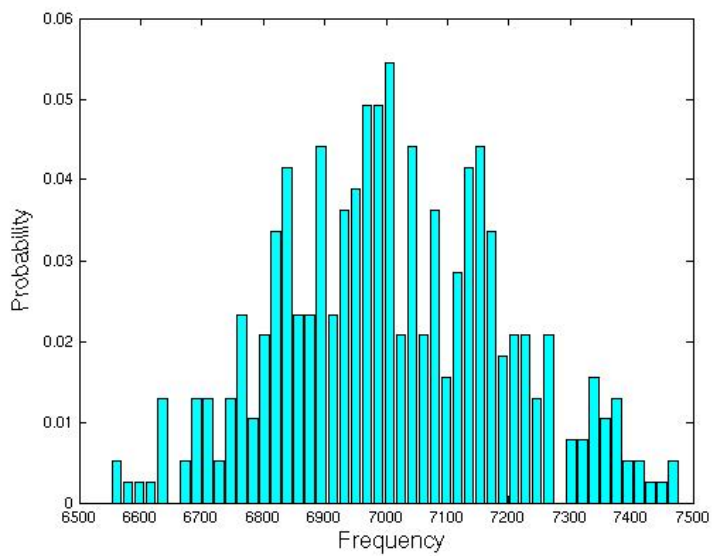

(c) Frequency Distribution.

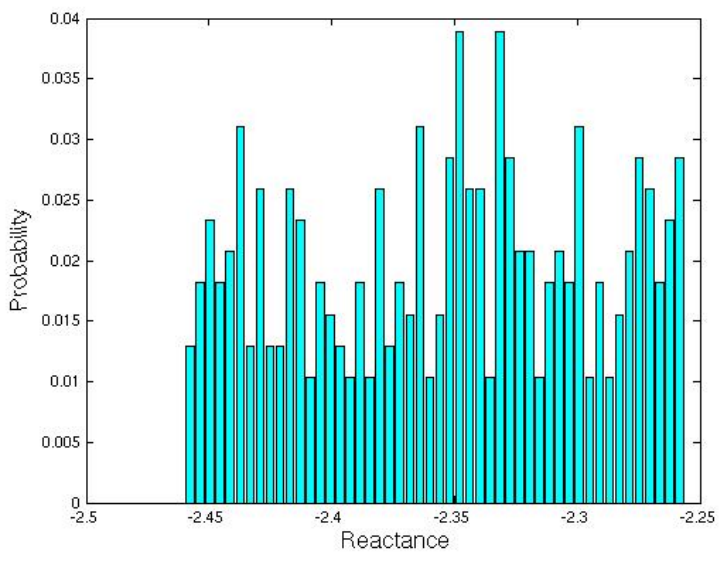

(b) Reactance Distribution.

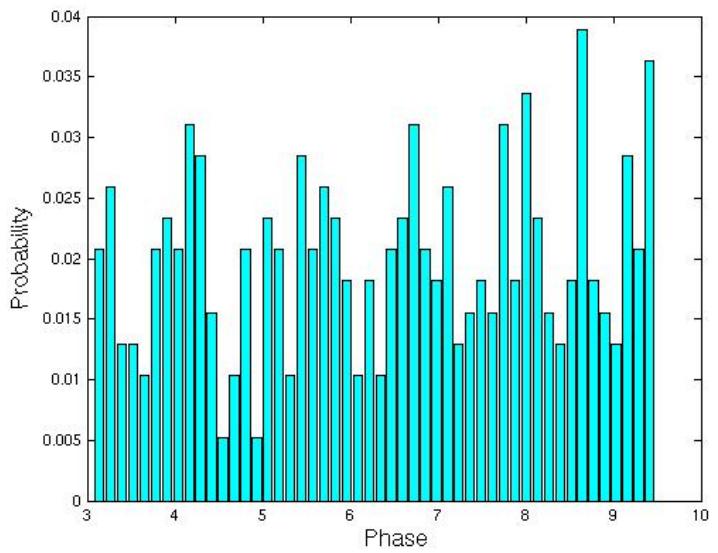

(d) First Mode Phase Distribution.

Figure 4. Probability distribution functions of variables for uniform liner Monte Carlo simulation.

The resulting probability density functions for the uniform and checkerboard liners are shown in Fig. 6. Note that the attenuations are negative in this figure. Larger negative numbers indicate greater attenuation. This is the opposite of the deterministic results. The figures show that the variance in the uniformly lined duct is smaller than that for the checkerboard lined duct. This is consistent with previous findings and indicates that the uniformly lined duct is less sensitive to uncertainties than is the checkerboard lined duct. The distribution for the checkerboard lined duct is bounded on the upper limit of attenuation by the deterministic value of -3.74 where as the distribution of the uniformly lined duct is not. The deterministic value, -2.28 , of the uniformly lined duct falls nearer the mean of the distribution. This result was unexpected and had 


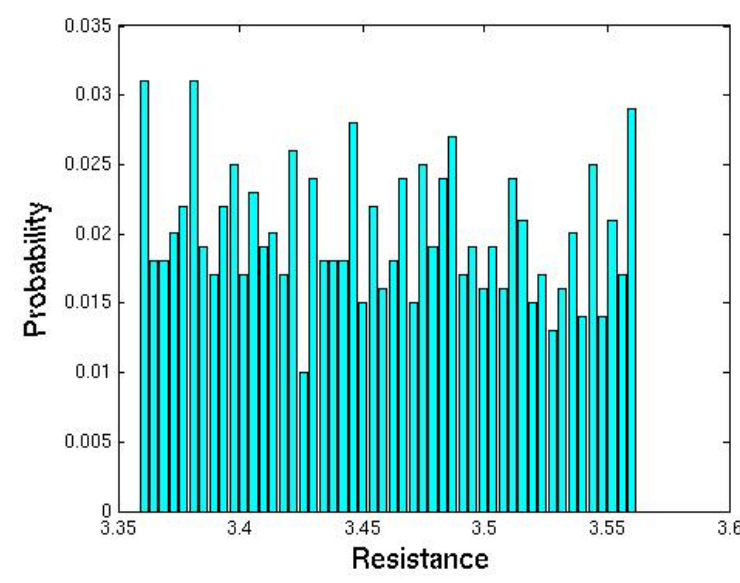

(a) First Segment Resistance Distribution.

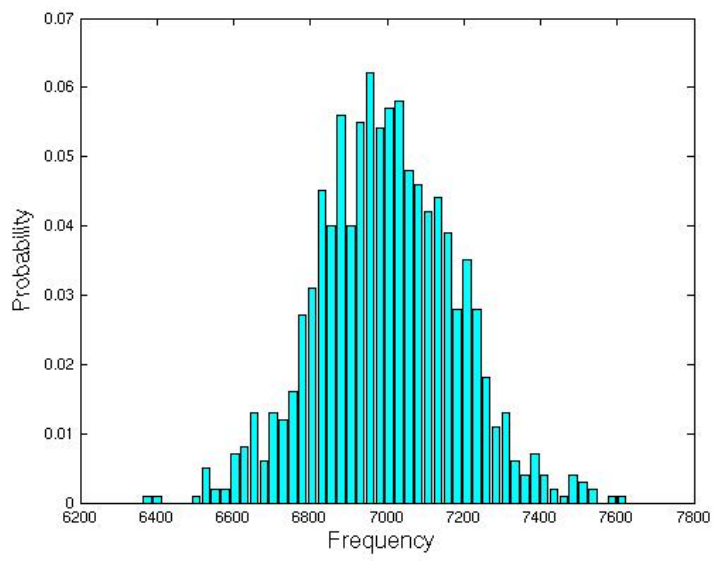

(c) Frequency Distribution.

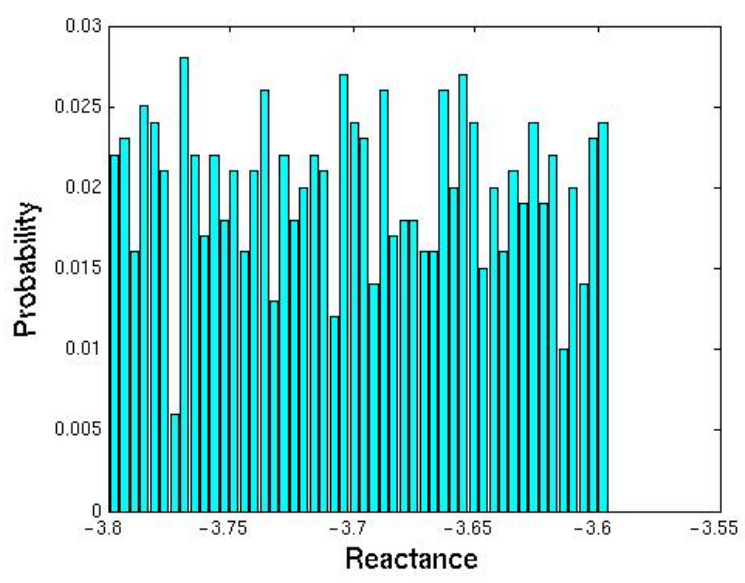

(b) First Segment Reactance Distribution.

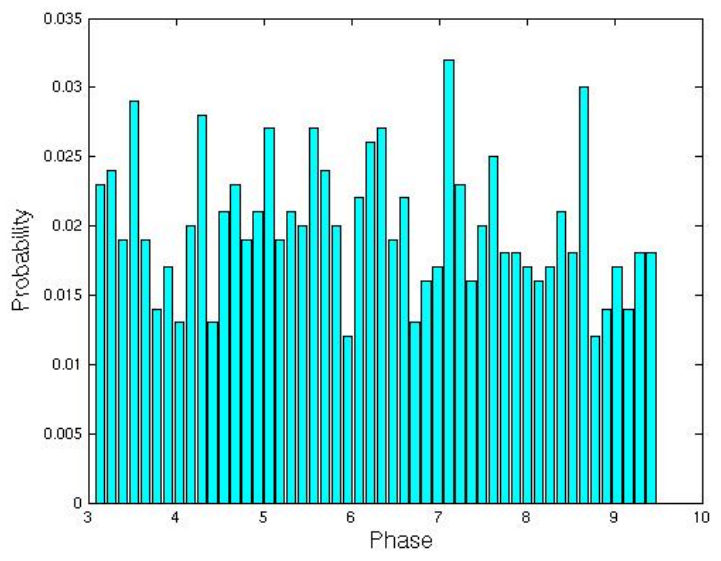

(d) First Mode Phase Distribution.

Figure 5. Probability distribution functions of select variables for segmented liner Monte Carlo simulation. 
not been seen until uncertainties in frequency and phase were included in the simulations. An examination of the raw data yielded little information or insight into the cause of the resulting distribution, however, the resulting distribution appears to be comprised of at least two distinct effects combining independently. The first appears to be a distribution comprising the right lobe of the resulting distribution and believed primarily due to uncertainties in source structure. The second is believed to be responsible for the left lobe and arising from the frequency distribution. The frequency distribution spans a range in which duct modes are being cut-on and cutoff and also in which the frequency versus liner attenuation curve may have a steep slope. This second effect is believed responsible for the attenuations greater than the deterministic attenuation. This discussion of the distribution is speculative but is consistent with anecdotal information.

The distribution of the attenuation of the checkerboard lined duct is characteristic of a highly tuned dynamic system. Performance drops off dramatically with even the slightest change in the operating conditions. This figure indicates that there is virtually no possibility of obtaining the $3.74 \mathrm{~dB}$ per $L / H$ of additional attenuation predicted by the deterministic analysis. This observation was consistent with previous studies and has been one reason why such segmented liners have not been considered viable.

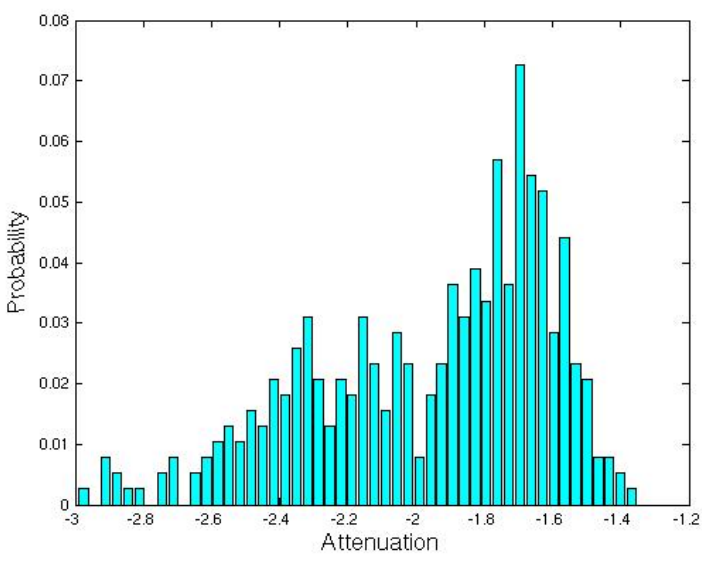

(a) Uniform liner.

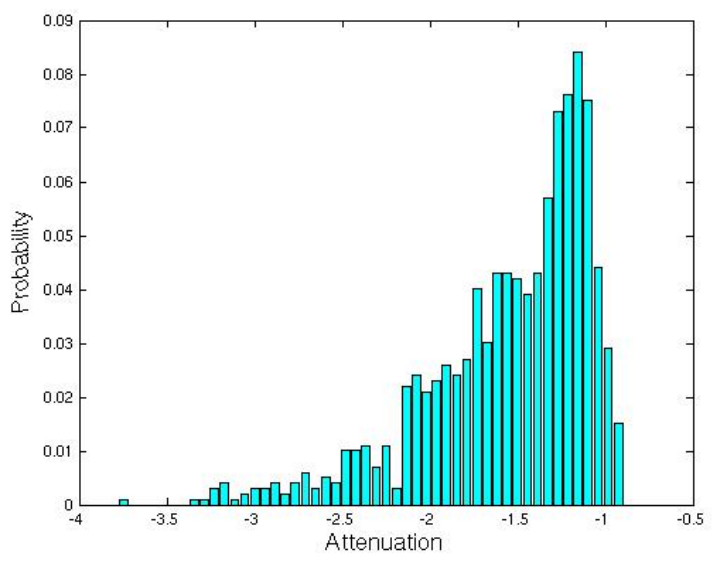

(b) Checkerboard liner.

Figure 6. Probability distribution functions of duct attenuation for checkerboard and uniform liners.

\section{Liner Performance with Uncertainty}

The probability that the checkerboard liner would outperform the uniform liner by an increment $\Delta$ is plotted in Fig. 7. The first and obvious thing to note is that this checkerboard liner has a less than 25 percent chance of outperforming the uniform liner under the range of conditions specified in the Monte Carlo simulations. The deterministic performance gain of approximately $1.5 \mathrm{~dB}$ per duct aspect ratio is virtually nonexistent when the influence of uncertainties are included in the assessment. This result is valid only for this liner configuration with these values of impedance at this frequency. The results from a deterministically better designed checkerboard liner could be substantially better. This result does not address the full performance of the liner over the full frequency range of previous investigations and, therefore, does not address whether the uniform liner or the checkerboard liner has better broadband characteristics.

\section{Discussion}

11 of 13 
The results of this investigation show the potential advantages and disadvantages of a checkerboard liner. The configuration of the checkerboard liner was determined by deterministic optimization and the assessment of its performance carried out by Monte Carlo simulation. The liner design variables were those of the DC component of flow resistance and liner depth. This investigation did not constrain these quantities to be physically realizable. This investigation, also, did not take into ac-

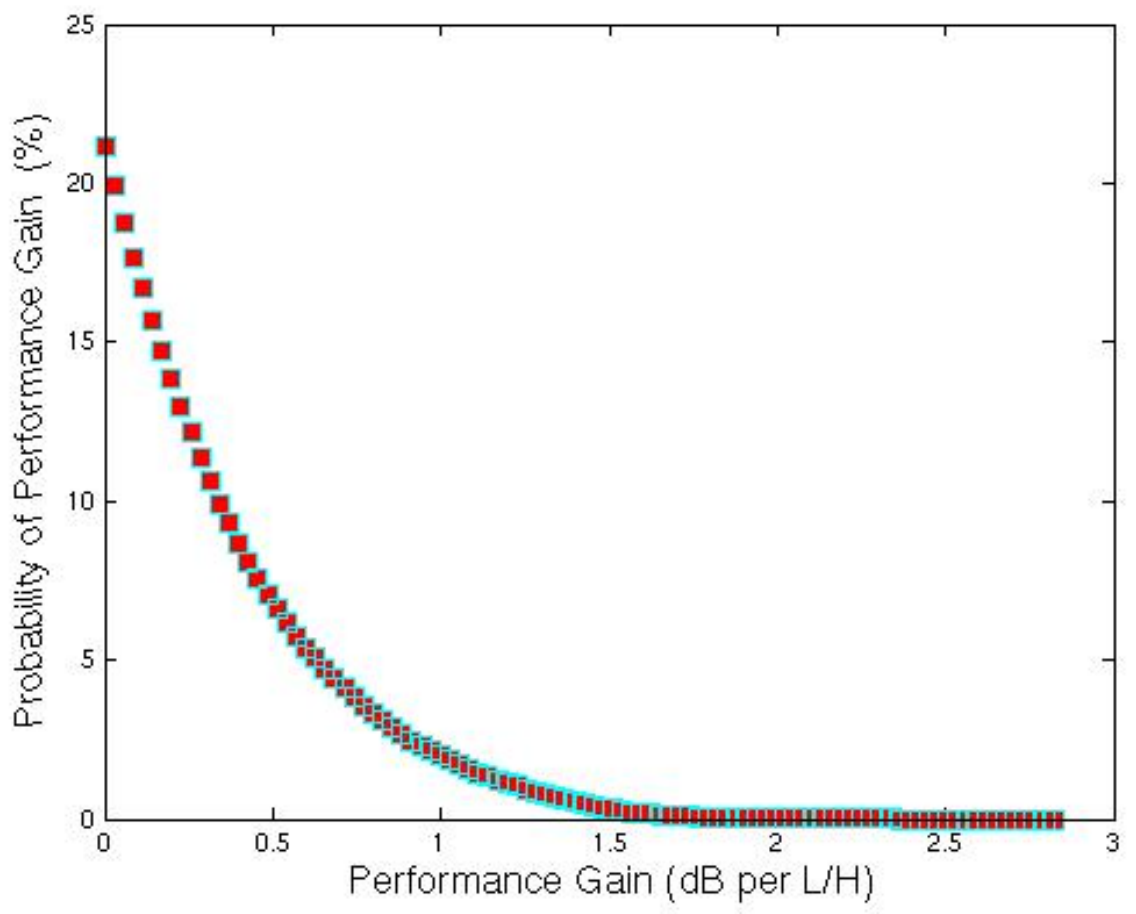
count the influence of the flow field and other characteristic dimensions of locally reacting duct liners. Results of this investigation did show that this checkerboard liner was unlikely to outperform a uniform liner under the assumptions of uncertainty in impedance, source phase, and frequency oscillations.

\section{References}

\footnotetext{
${ }^{1}$ Mani, R., "General Electric Co. (Schenectady, New York)," Acoustic Duct with Peripherally Segmented Acoustic Treatment, U.S. Patent 3,937,590 (1976).

${ }^{2}$ Howe, M. S., "The Attenuation of Sound in a Randomly Lined Duct," Journal of Sound and Vibration, Vol. 87, No. 1, 1983, pp. 83-103.

${ }^{3}$ Watson, W. R., Jones, M. G., Parrott, T. L., and Sobieski, J., "A Method for Optimizing Non-Axisymmetric Liners for Multi-Modal Sound Sources," Proceedings of the 8th AIAA/CEAS Aeroacoustics Conference \& Exhibit. AIAA 2002-2516, June 2002.

${ }^{4}$ Watson, W. R., Robinson, J. H., Jones, M. G., Parrott, T. L., and Sobieski, J., "Design and Attenuation Properties of Periodic Checkerboard Liners," Proceedings of the 9th AIAA/CEAS Aeroacoustics Conference \& Exhibit. AIAA 2003-3309, May 2003.

${ }^{5}$ Watson, W. R., Robinson, J. H., Jones, M. G., and Parrott, T. L., "Computational Study of Optimum and Off-Design Performance of Checkerboard Liners," Proceeding of the 10th AIAA/CEAS Aeroacoustics Conference \& Exhibit. AIAA 20043030, May 2004.

${ }^{6}$ Motsinger, R. D., Kraft, R. E., Paas, J. E., and Gahn, B. M., "Analytical and Experimental Results of Some Segmented Liner Acoustic Performance Studies for High Mach Number Inlets," Tech. Rep. NASA CR-2882, June 1974.

${ }^{7}$ Kraft, R. E., Theory and Measurement of Acoustic Wave Propagation in Multi-Segmented Rectangular Flow Ducts, Ph.D. thesis, Department of Mechanical Engineering, University of Cincinnati, Cincinnati, Ohio, 1976.

${ }^{8}$ Lester, H. C. and Posey, J. W., "Optimum One-Section and Two-Section Circular Sound Absorbing Duct Liners for Plane-Wave and Monopole Sources Without Flow," Tech. Rep. NASA TN D-8348, 1976.
} 
${ }^{9}$ Baumeister, K. J., "Optimized Multisectioned Acoustic Liners," Proceeding of the 17th AIAA Aerospace Sciences Meeting. AIAA 1979-1829, Jan. 1979.

${ }^{10}$ Actran User's Manual, Free Field Technologies, Louvain-la-Neuve, Belgium, 2004.

${ }^{11}$ Gallman, J. M. and Kunze, R. K., "Grazing Flow Acoustic Impedance Testing for the NASA AST Program," Proceeding of the 8th AIAA/CEAS Aeroacoustics Conference \& Exhibit. AIAA 2002-2447, June 2002.

${ }^{12}$ Engenious Software Company, iSIGHT Reference Guide, Version 6.0, Addison-Wesley Inc., New York, NY, 2001. 